\title{
SELETIVIDADE DO EXTRATO ETANÓLICO DE Annona muricata (L. 1753) (ANNONACEAE) E DE ABAMECTINA AO ÁCARO PREDADOR Amblyseius aerialis (MUMA, 1955) (ACARI: PHYTOSEIIDAE)
}

\author{
Anilde da Graça Sousa Maciel', Márcia Daniela dos Santos ${ }^{1}$, Roseane Cristina Predes Trindade², Adriana \\ Guimarães Duarte ${ }^{2}$
}

\begin{abstract}
${ }^{1}$ Doutoranda em Proteção de Plantas, Centro de Ciências Agrárias, Universidade Federal de Alagoas, BR 104 Norte, Km 85, Rio Largo, AL. CEP 57.000-100.

${ }^{2}$ Professora, Centro de Ciências Agrárias, Universidade Federal de Alagoas, BR 104 Norte, Km 85, Rio Largo, AL. CEP 57.000-100.
\end{abstract}

*Autor para correspondência: Anilde da Graça Sousa Maciel, maciel_anilde@hotmail.com

\begin{abstract}
RESUMO: O ácaro Amblyseius aerialis (Muma, 1955) (Acari: Phytoseiidae) se destaca pela importância como inimigo natural de ácaros-praga, como Tetranychus urticae (Koch, 1836) (Acari: Tetranychidae), de grande relevância em diversas culturas agrícolas. Além do uso de acaricidas químicos para o controle dessa praga, trabalhos vêm sendo realizados na busca de acaricidas botânicos. As anonáceas apresentam substâncias com potencial acaricida, entre elas, as acetogeninas. No entanto, é importante estudar 0 efeito de acaricidas sobre os inimigos naturais. Portanto, o trabalho teve como objetivo avaliar a seletividade da abamectina e do extrato etanólico de sementes de Annona muricata ao ácaro A. aerialis. Discos de folhas de Canavalia ensiformis L. DC (Fabaceae) foram pulverizados com os produtos. Em seguida, foram transferidas cinco fêmeas de $A$. aerialis para cada disco e T. urticae foi oferecido como alimento. Cada teste teve duração de oito dias, com a contagem diária das fêmeas vivas e do número de ovos postos. Os resultados mostraram que a abamectina apresentou efeito nocivo ao ácaro $A$. aerialis, com $100 \%$ de mortalidade, enquanto o extrato etanólico de semente de $A$. muricata foi inócuo $(E \%=24,14)$ e apresentou efeito reduzido na reprodução.
\end{abstract}

PALAVRAS-CHAVE: inimigo natural, acaricida botânico, acaricida químico.

\section{SELECTIVITY OF ETHANOLIC EXTRACT OF Annona muricata (L. 1753) (ANNONACEAE) AND ABAMECTIN TO THE PREDATOR MITE Amblyseius aerialis (Muma, 1955) (ACARI: PHYTOSEIIDAE)}

ABSTRACT: The mite Amblyseius aerialis (Muma, 1955) (Acari: Phytoseiidae) stands out by its importance as natural enemy of pest mites, such as Tetranychus urticae (Koch, 1836) (Acari: Tetranychidae), very important pest in many crops. Besides the use of chemical acaricides to control this pest, studies have been conducted in search of botanical acaricides. Annonaceous plants have substances with acaricidal potential, among them, the acetogenins. However, it is essential to study the effect of acaricides on natural enemies. Therefore, the study aimed at evaluating the selectivity of abamectin and ethanolic extract of Annona muricata seeds to the mite $A$. aerialis. Leaf discs of Canavalia ensiformis L. DC (Fabaceae) were sprayed with the products. Then, five females of $A$. aerialis were transferred to each disc and $T$. urticae was offered as food. Each test lasted for eight days, with the daily count of living females and eggs laid. The results showed that the abamectin caused harmful effect to the mite $A$. aerialis, with $100 \%$ mortality, while the ethanolic extract of $A$. muricata seeds was innocuous $(E=24.14 \%)$ and presented reduced effect on reproduction.

KEYWORDS: natural enemy, botanical acaricide, chemical acaricide.

\section{INTRODUÇÃO}

A família de ácaros predadores Phytoseiidae se destaca como a de maior importância em programas de controle biológico. Caracterizam-se por apresentar pernas longas e movimentação rápida, e podem ser encontrados nas plantas e no solo, mas não são predominantes neste ambiente. São eficazes no controle de ácaros da família Tetranychidae e, por isso, são os ácaros predadores mais estudados (Moraes e Flechtmann, 2008). 
A espécie Amblyseius aerialis (Muma, 1955) pode contribuir na regulação natural de ácaros-praga, devido ao seu hábito alimentar generalista e ampla distribuição, sendo comumente encontrada no Brasil em frutíferas, roseiras e fragmentos florestais (Forero et al., 2008; Noronha et al., 2010; Silva et al., 2010; Bobot et al., 2011).

A eficiência de Amblyseius sp. foi relatada no controle biológico dos ácaros fitófagos Tetranychus urticae (Koch, 1836) (Tetranychidae) e Brevipalpus phoenicis (Geijskes, 1939) (Tenuipalpidae) (Forero et al., 2008).

O ácaro T. urticae é considerado uma praga de grande importância em diversas culturas agrícolas, sua ocorrência é registrada para mais de 1.100 espécies, pertencentes a 140 famílias botânicas, incluindo frutíferas, olerícolas e ornamentais (Moraes e Flechtmann, 2008).

A principal estratégia adotada para o manejo de T. urticae é o uso de acaricidas químicos, dentre estes 0 Abamectin Nortox ${ }^{\circledR}$ que é indicado para várias culturas no Brasil e caracterizado por ter ação de contato e de ingestão (Agrofit, 2015).Como alternativa ao controle químico, trabalhos vêm sendo realizados na busca de acaricidas botânicos que apresentem eficiência e que sejam menos agressivos ao meio ambiente, ao homem e a organismos benéficos (Castagnoli et al., 2002; Mourão et al., 2004; Martinez-Villar et al., 2005; Esteves Filho et al., 2013; Krinski et al., 2014).

As plantas da família Annonaceae apresentam em sua composição várias substâncias com potencial inseticida/acaricida, entre elas, as acetogeninas que são as mais importantes por apresentarem bioatividade contra diversas espécies (Alali et al., 1999). 0 extrato etanólico de sementes Annona muricata (L.1753) foi eficiente no controle de $T$. urticae, com $\mathrm{CL}_{99}$ estimada em 12,07 mg/mL, além de apresentar efeito repelente e toxicidade sobre os ovos, segundo Maciel et al. (2015). No entanto, é fundamental estudar o efeito de acaricidas aos inimigos naturais.

0 uso de ácaros predadores, como os da espécie $A$. aerialis, associado ao uso de acaricidas botânicos seletivos pode ser uma alternativa viável para o controle de T. urticae, minimizando os riscos de presença de resíduos de agrotóxicos nos alimentos e impactos ao ambiente (Veronez et al., 2012). Com base no exposto, o trabalho teve como objetivo avaliar a seletividade do acaricida Abamectin
Norto ${ }^{\circledR}$ e do extrato etanólico de A. muricata ao ácaro predador $A$. aerialis.

\section{MATERIAL E MÉTODOS}

O trabalho foi realizado no Laboratório de Entomologia: Controle alternativo de pragas do Centro de Ciências Agrárias da Universidade Federal de Alagoas - CECA/UFAL, à temperatura de $26 \pm 1^{\circ} \mathrm{C}$, umidade relativa de $60 \pm 10 \%$ e fotofase de $12 \mathrm{~h}$.

\section{Criação de Tetranychus urticae}

Os ácaros foram provenientes de criação mantida em laboratório, em plantas de feijão-de-porco (Canavalia ensiformis L. DC) (Fabaceae) cultivadas em vasos de polietileno com capacidade de $5 \mathrm{~L}$, contendo uma mistura de solo, bagaço de coco, adubo 10-10-10 e esterco bovino. Os vasos permaneceram em gaiolas de criação de 0,50 x 0,50 × 0,50 m cobertas com tela antiafídica e foram substituídos por novas plantas assim que a população iniciava a formação de teias para se dispersar.

\section{Criação de Amblyseius aerialis}

Os ácaros foram oriundos de criação mantida em laboratório, sobre discos de folhas de feijão-de-porco (5,0 cm de diâmetro), segundo a metodologia descrita por Reis e Alves (1997). Os discos foram colocados para flutuar sobre água destilada em placas de Petri sem tampa (8,5 cm de diâmetro). Cada disco continha um orifício central para a passagem de um alfinete, fixado com a cabeça voltada para o fundo da placa com auxílio de cola à base de silicone. Foi colocado sobre os discos um pedaço de plástico transparente dobrado em forma de pirâmide, para servir de abrigo e local de postura. Como fonte de alimentos para os predadores, utilizou-se $T$. urticae em abundância em todas as fases de desenvolvimento. Os ácaros predadores eram transferidos para novos discos infestados com T. urticae, com 0 auxílio de um pincel de cerdas finas, assim que a fonte de alimento acabava.

\section{Produtos utilizados}

Foi utilizado o acaricida Abamectin Nortox ${ }^{\circledR}$ (CE $18 \mathrm{~g} / \mathrm{L}$; Nortox S.A. - Arapongas) na dosagem comercial (100 mL/100 L) e o extrato etanólico de sementes de A. muricata $(12,07 \mathrm{mg} / \mathrm{mL})$ preparado no Laboratório 
de Pesquisa em Recursos Naturais da UFAL. Para o preparo do extrato, sementes de $A$. muricata foram colocadas em sacos de papel Kraft e levadas para secagem em estufa com circulação de ar à temperatura de $50^{\circ} \mathrm{C}$ por sete dias. Em seguida, foram trituradas em moinho de facas tipo Wiley para a obtenção do pó.

Foram utilizados $6 \mathrm{~kg}$ do pó das sementes, submetidos à extração a frio em percolador de aço inoxidável, primeiramente com hexano $\left[\mathrm{CH}_{3}\left(\mathrm{CH}_{2}\right) 4 \mathrm{CH}_{3}\right]$ $(8 \mathrm{~L})$, durante 24 horas, depois sobre a torta resultante dessa extração acrescentou-se etanol $\left(\mathrm{CH}_{3} \mathrm{CH}_{2} \mathrm{OH}\right)$ para a extração a frio por três vezes durante 72 horas. $\mathrm{Na}$ primeira, segunda e terceira extrações, utilizaramse 6,0; 6,6 e 5,0 L de etanol, respectivamente. 0 material resultante das extrações foi filtrado e posteriormente concentrado em rotaevaporador a $50^{\circ} \mathrm{C}$, sob pressão reduzida. 0 resíduo concentrado obtido da fração etanólica foi colocado em frasco de vidro hermeticamente fechado e armazenado em temperatura ambiente.

\section{Bioensaio}

Discos de folhas de feijão-de-porco de $5,0 \mathrm{~cm}$ de diâmetro foram pulverizados com os respectivos produtos, utilizando-se torre de Potter (Burkard, Rickmansworth, UK), a uma pressão de $5 \mathrm{psi} / \mathrm{pol}^{2}$ com um volume de calda de $2,3 \mathrm{~mL}$, correspondendo a um depósito de 1,9 $\pm 0,37 \mathrm{mg} / \mathrm{cm}^{2}$. Esta quantidade aplicada está de acordo com a recomendação do IOBC/ WPRS (Reis et al., 1998).

Em seguida, os discos foram colocados para secar sobre papel toalha à temperatura ambiente durante uma hora e colocados para flutuar sobre água, no interior de placas de Petri ( $8,5 \mathrm{~cm}$ de diâmetro), conforme metodologia descrita por Reis e Alves (1997). Posteriormente, cinco fêmeas de $A$. aerialis fecundadas foram transferidas para cada disco, e $T$. urticae para servir de alimento. As presas foram ofertadas na relação predador:presa (1:5) e repostas a cada 24 horas. Foram utilizadas 12 repetições por produto.

Cada teste teve a duração de oito dias, com a contagem diária das fêmeas vivas e do número de ovos postos. A avaliação da viabilidade dos ovos ocorreu por cinco dias, tempo suficiente para a eclosão das larvas nas condições do bioensaio.

$O$ efeito na reprodução $\left(E_{r}\right)$ foi obtido pela divisão da produção média de ovos viáveis nos tratamentos pela produção média de ovos viáveis na testemunha $\left(E_{r}=\right.$ Rtratamento/Rtestemunha). A produção média de ovos $(R)$ foi obtida através da relação: $R=$ número de ovos viáveis /número de fêmeas vivas. $O$ efeito total ou adverso $(\mathrm{E} \%)$ do produto foi calculado levando-se em consideração a mortalidade das fêmeas no tratamento, corrigida em função da mortalidade na testemunha, e o efeito na reprodução, através da fórmula: $E \%=100 \%-\left(100 \%-M_{c}\right)$ $x E_{r}$, onde $M_{c}=$ mortalidade corrigida (Henderson e Tilton, 1955) e $E_{r}=$ efeito na reprodução (Hassan et al., 1985; Overmeer, 1988; Bakker et al., 1992).

Os efeitos totais, encontrados para cada produto foram classificados nas classes de 1 a 4 , conforme critérios estabelecidos pela IOBC MPRS para enquadrar produtos fitossanitários, quanto ao efeito adverso causado a organismos benéficos em testes de laboratório (Bakker et al., 1992; Hassan et al., 1994), sendo: classe $1=\mathrm{E}<30 \%$ (inócuo ou não nocivo); classe $2=30 \%<\mathrm{E}<79 \%$ (levemente nocivo); classe $3=80 \%<E<99 \%$ (moderadamente nocivo) $\mathrm{e}$; classe $4=\mathrm{E}>99 \%$ (nocivo).

\section{RESULTADOS E DISCUSSÃO}

A abamectina mostrou-se nociva ao ácaro $A$. aerialis, causando mortalidade de 100\% (Tabela 1). Esta mortalidade foi observada logo nas primeiras 48 horas e dessa forma, 0 efeito na reprodução não pode ser avaliado.

Tabela 1. Toxicidade de abamectina e de extrato etanólico de sementes de Annona muricata sobre fêmeas adultas de Amblyseius aerialis, em folhas de feijão-de-porco.

\begin{tabular}{lcccc}
\hline \multicolumn{1}{c}{ Produto } & $\mathrm{M}_{\mathrm{c}}^{\mathrm{a}}(\%)$ & $\mathrm{E}_{\mathrm{r}}^{\mathrm{b}}$ & $\mathrm{E}^{\mathrm{c}}{ }^{\mathrm{c}}$ & Classe $^{\mathrm{d}}$ \\
\hline Abamectin Nortox $^{\circledR}$ & 100,00 & - & 100,00 & 4 \\
Extrato etanólico de Annona muricata & 22,91 & 0,98 & 24,14 & 1 \\
\hline
\end{tabular}

${ }^{a} M_{c}=$ Mortalidade corrigida; ${ }^{b} E_{r}=$ Efeito na reprodução; ${ }^{c} E \%$ = Efeito total; ${ }^{d}$ Classe de toxicidade segundo IOBC/ WPRS. 
Resultados semelhantes, nos quais abamectina (Vertimec ${ }^{\circledR} 1.8 \%$ CE) causou mortalidade superior a $90 \%$ ao ácaro predador Neoseiulus californicus (McGregor, 1954) (Acari: Phytoseiidae) em morangueiro e de 79,6\% em citros foram observados por Sato et al. (2002) e por Silva e Oliveira (2006), respectivamente.

Sato et al. (2011) constataram que abamectina (Vertimec ${ }^{\circledR 1.8 \% ~ C E) ~ c a u s o u ~ e f e i t o s ~ n e g a t i v o s ~ s o b r e ~}$ $N$. californicus, com reduções drásticas (> 96\%) no número de ovos postos e valores negativos da taxa de crescimento populacional com uma tendência para causar a extinção do ácaro.

A elevada toxicidade da abamectina também foi observada para ácaros predadores pertencentes à família Stigmaeidae, como Agistemus brasiliensis Matioli, Ueckermann \& Oliveira, 2002 (Silva et al., 2009; Fuzita et al., 2014) e A. industani Gonzalez-Rodrigues, 1965 (Childerg et al., 2001).

0 extrato etanólico de $A$. muricata na concentração de $12,07 \mathrm{mg} / \mathrm{mL}$, foi inócuo ao ácaro $A$. aerialis $(E \%=24,14)$, apresentou efeito reduzido na reprodução, com o $E_{r}$ ligeiramente inferior a $1\left(E_{r}=\right.$ 0,98) (Tabela 1), e não afetou a viabilidade dos ovos.

Outros extratos vegetais também se mostraram seletivos a ácaros predadores. 0 extrato de folhas e sementes de nim, Azadirachta indica A. Juss. (Meliaceae), foi seletivo ao ácaro predador Iphiseiodes zuluagai (Denmark \& Muma, 1972) (Acari: Phytoseiidae) (Mourão et al., 2004). Da mesma forma, foi observada a baixa mortalidade de ácaros fitoseídeos, quando tratados com produtos formulados à base de nim. No tratamento com Neemseto ${ }^{\circledR}$ a $1,0 \%$, verificou-se mortalidade de $6,3 \%$ e 3,8\% para Euseius alatus De Leon, 1966 e Phytoseiulus macropilis (Banks, 1904), respectivamente (Brito et al., 2006).

Segundo, Schlesener et al. (2013), o ácaro $N$. californicus apresentou mortalidade de $6,8 \%$ no tratamento com Azamax $^{\circledR}$ e P. macropilis $5,5 \%$ de mortalidade no tratamento com Neemseto ${ }^{\circledR}$, ao final de 168 horas após a aplicação. Para a mesma espécie, a mortalidade foi de $25 \%$, quando utilizado o Natuneem ${ }^{\circledR}$ (Veronez et al., 2012).

Produtos formulados à base de nim são os mais estudados, desta forma, pouco se sabe sobre os demais produtos de origem botânica e seus efeitos sobre ácaros predadores. Geralmente são mais seletivos aos inimigos naturais, se comparados com os acaricidas químicos, devido a características inerentes aos extratos e/ou metabolização enzimática (Mourão et al., 2004; Soto et al., 2011).

Com base nos resultados apresentados no presente trabalho, conclui-se que a abamectina apresentou efeito nocivo ao ácaro $A$. aerialis e a utilização do extrato etanólico de $A$. muricata é uma boa alternativa no manejo integrado, visto que foi inócuo ao ácaro predador $A$. aerialis.

\section{REFERÊNCIAS BIBLIOGRÁFICAS}

Agrofit (2015) Sistema de Agrotóxicos Fitossanitários, Ministério da Agricultura, Pecuária e Abastecimento. URL (http: extranet.agricultura.gov.br/agrofit_cons/ principal_agrofit_cons.) 24 Jan 2015.

Alali, F.Q.; Liu, X.X.; McLaughlin, J.L. Annonaceous acetogenins: recent progress. Journal of Natural Products, 1999, 62, 504-540.

Bakker, F.; Grove, A.; Blumel, S.; Calis, J.; Oomen, $P$. Side-effect tests for phytoseiids and their rearing methods. IOBC/WPRS Bull, 1992, 15, 3, 61-81.

Bobot, T.E.; Franklin, E.; Navia, D.; Gasnier, T.R.J.; Lofego, A.C.; Oliveira, B.M. Mites (Arachnida, Acari) on Citrus sinensis L. Osbeck orange trees in the state of Amazonas, Northern Brazil. Acta Amazonica, 2011, 41, 4, 557-566.

Brito, H.M.; Gondim Junior, M.G.C.; Oliveira, J.V.; Câmara, C.A.G. Toxicidade de Natuneem sobre Tetranychus urticae Koch (Acari: Tetranychidae) e ácaros predadores da família Phytoseiidae. Ciência e Agrotecnologia, 2006, 30, 4, 685-691.

Castagnoli, M.; Angeli, G.; Liguori, M.; Forti, D.; Simoni, $S$. Side effects of botanical insecticides on predatory mite Amblyseius andersoni (Chant). Journal of Pest Science, 2002, 75, 5, 122-127.

Childers, C.C.; Villanueva, R.; Aguilar, H.; Chewning, R.; Michaud, J.P. Comparative residual toxicities of pesticides to the predator Agistemus industani (Acari: Stigmaeidae) on citrus in Florida. Experimental and Applied Acarology, 2001, 25, 6, 461- 474. 
Esteves Filho, A.B.; Oliveira, J.V.; Torres, J.B.; Matos, C.H.C. Toxicidade de espiromesifeno e acaricidas naturais para Tetranychus urticae Koch e compatibilidade com Phytoseiulus macropilis (Banks). Semina: Ciências Agrárias, 2013, 34, 6, 2675-2686

Forero, G.; Rodríguez, M.; Cantor, F.; Rodríguez, D.; Cure, J.R. Criterios para el manejo de Tetranychus urticae Koch (Acari: Tetranychidae) con el ácaro depredador Amblyseius (Neoseiulus) sp. (Acari: Phytoseiidae) em cultivos de rosas. Agronomía Colombiana, 2008, 26, 1, 1-17.

Fuzita, A.T.; Sato, M.E.; Silva, M.Z.; Nicastro, R.L.; Mendonça, M.J.C. Comparação da sensibilidade do ácaro-praga Brevipalpus phoenicis e do predador Agistemus brasiliensis a agroquímicos. Coffee Science, 2014, 9, 1, 102-109.

Hassan, S.A.; Bigle, F.; Blaisinger, P.; Bogenshutz, H.; Brun, J.; Chiverton, P.; Dickler, E.; Easterbrook, M.A.; Edwards, P.J.; Englert, W.D.; Firth, S.I.; Huang, P.; Inglesfield, D.; Klingauf, F.; Kuhner, C.; Overmeer, W.P.J.; Plevoets, P.; Reboulet, J.N.; Rieckmann, W.; Samsoe-Petersen, L.; Shires, S.W.; Staubli, A.; Stevenson, J.; Tuset, J.J.; Vanwetswinkel, G.; Vanzon, Q. Standard method to test the side-effects of pesticides on natural enemies of insects and mites developed by the IOBC/WPRS - working group "Pesticides and Beneficial Organisms". EPPO Bulletin, 1985, 15, 214255.

Hassan, S.A; Bigle, F.; Bogenschutz, H.; Boller, E.; Brun, J.; Chiverton, P.; Edwards, P.J.; Mansour, F.; Naton, E.; Oomen, P.A.; Overmeer, W.P.J.; Polgar, L.; Rieckmann, W.; Samsoe-Petersen, L.; Staubli, A.; Sterk, G.; Tavares, K.; Tuset, J.J.; Viggiani, G.; Vivas, A.G. Results of the sixth joint pesticide testing programme of the IOBC / WPRS - working group pesticides and beneficial organisms. Entomophaga, 1994, 39, 1, 109119.

Henderson, C.F.; Tilton, E.W. Tests with acaricides against the brown wheat mite. Journal of Economic Entomology, 1955, 63, 1536- 1539.

Krinski, D.; Massaroli, A.; Machado, M. Potencial inseticida de plantas da família Annonaceae. Revista Brasileira de Fruticultura, 2014, 36, 225-242.
Maciel, A.G.S.; Rodrigues, J.S.; Trindade, R.C.P.; Silva, E.S.; Sant'Ana, A.E.G.; Lemos, E.E.P. Effect of Annona muricata L. (1753) (Annonaceae) seeds extracts on Tetranychus urticae (Koch, 1836) (Acari: Tetranychidae). African Journal of Agricultural Research, 2015, 10, 48, 4370-4375.

Martínez-Villar, E.; Sáenz-de-Cabezón, F.J.; MorenoGrijalba, F.; Marco, V.; Pérez-Moreno, I. Effects of azadirachtin on the two-spotted spider mite, Tetranychus urticae (Acari: Tetranychidae). Experimental and Applied Acarology, 2015, 35, 3, 215-222.

Moraes, G.J.; Flechtmann, C.H.W. Manual de Acarologia. Acarologia básica e ácaros de plantas cultivadas no Brasil. Ribeirão Preto: Holos, 2008, 308 p.

Mourão, S.A.; Silva, J.C.T.; Guedes, R.N.C.; Venzon, M.; Jham, G.N.; Oliveira, C.L.; Zanucio, J.C. Seletividade de extratos de nim (Azadirachta indica A. Juss.) ao ácaro predador Iphiseiodes zuluagai Denmark \& Muma (Acari: Phytoseiidae). Neotropical Entomology, 2004, 33, 5, 613-617.

Noronha, A.C.S.; Argolo, P.S.; Boaventura, V.J.; Ritzinger, R. Ácaros (Acari) em mudas de aceroleira. Boletín de Sanidad Vegetal Plagas, 2010, 36, 189-195.

Reis P.R; Chiavegato, L.G.; Moraes, G.J.; Alves, E.B.; Sousa, E. O. Seletividade de agroquímicos ao ácaro predador Iphiseiodes zuluagai Denmark \& Muma (Acari: Phytoseiidae). Anais da Sociedade Entomológica do Brasil, 1998, 27, 265-274.

Reis, P.R.; Alves, E.B. Criação do ácaro predador Iphiseiodes zuluagai Denmark Muma (Acari: Phytoseiidae) em laboratório. Anais da Sociedade Entomológica do Brasil, 1997, 26, 565-568.

Sato, M.E.; Silva, M.Z.; Raga, A.; Cangani, K.G.; Veronez, B.; Nicastro, R.L. Spiromesifen toxicity to the spider mite Tetranychus urticae and selectivity to the predator Neoseiulus californicus. Phytoparasitica, 2011, 39, 437-445.

Sato, M.E.; Silva, M.; Gonçalves, L.R.; Souza Filho, M.F.; Raga, A. Toxicidade diferencial de agroquímicos a Neoseiulus californicus (McGregor) (Acari: Phytoseiidae) e Tetranychus urticae Koch 
(Acari: Tetranychidae) em morangueiro. Neotropical Entomology, 2002, 31, 3, 449-456.

Schlesener, D.C.H.; Duarte, A.F.; Guerrero, M.F.C; Cunha, U.S.; Nava, D.E. Efeitos do nim sobre Tetranychus urticae Koch (Acari: Tetranychidae) e os predadores Phytoseiulus macropilis (Banks) e Neoseiulus californicus (McGregor) (Acari: Phytoseiidae). Revista Brasileira de Fruticultura, 2013, $35,1,59-66$.

Silva, E.A.; Reis, P.R.; Zacarias, M.S. Fitoseídeos (Acari: Phytoseiidae) associados a cafezais e fragmentos florestais vizinhos. Ciência e Agrotecnologia, 2010, 34, 5, 1146-1153.

Silva, M.Z.; Oliveira, C.A.L. Seletividade de alguns agrotóxicos em uso na citricultura ao ácaro predador Neoseiulus californicus (McGregor) (Acari: Phytoseiidae). Revista Brasileira de Fruticultura, 2006, 28, 2, 205-208.
Silva, M.Z.; Oliveira, C.A.L.; Sato, M.E. Seletividade de produtos fitossanitários sobre 0 ácaro predador Agistemus brasiliensis Matioli, Ueckermann \& Oliveira (Acari: Stigmaeidae). Revista Brasileira de Fruticultura, 2009, 31, 2, 388-396.

Soto, A.; Oliveira, H.G.; Pallini, A. Integración de control biológico y de productos alternativos contra Tetranychus urticae (Acari: Tetranychidae). Revista U.D.C.A. Actualidad \& Divulgación Científica, 2011, 14, 1, $23-29$

Veronez, B.; Sato, M.E.; Nicastro, R.L. Toxicidade de compostos sintéticos e naturais sobre Tetranychus urticae e o predador Phytoseiulus macropilis. Pesquisa Agropecuária Brasileira, 2012, 47, 4, 511-518. 